stören aber wie bei diesem die quantitative Acetonbestimmung, denn je nach den Lichtverhältnissen oder der Luftmenge im Apparat können die Werte beträchtlich schwanken. Wie bei der Bestimmung des Acetons mit Salicylaldehyd müssen daher Tageslicht und Luft vom Vanillin-KOH-Reagenz ferngehalten werden, indem man bei künstlicher Beleuchtung und in Stickstoffatmosphäre arbeitet. Die Löslichkeit des Vanillins in Wasser und in Alkali ist aber zu klein, als daß damit die Extinktionen erreicht werden können, die man mit methanolhaltiger Vanillinlösung oder mit einer wäßrigen Salicylaldehydlösung erzielen kann. In $0,1 \mathrm{~m} l$ Blut lassen sich daher die physiologischerweise vorkommenden Acetessigsäurekonzentrationen nicht mit einer wäßrigen, alkalischen Vanillinlösung bestimmen. Mit einer entsprechend größeren Blutmenge ist die Mikrokonvektionsmethode von BAHNER nicht mehr ohne weiteres durchführbar.

Vanillin ist in Methanol sehr gut löslich (bis 50\%). Methanol ermöglicht auch höhere $\mathrm{KOH}-$ Konzentrationen, die wegen der Farbvertiefung erwünscht sind, aber es hat verschiedene Nacbteile:

1. Methanol schwächt in höheren Konzentrationen die Farbe des Divanillalacetons ab (Abb. 2);

2. die Extinktionen des Divanillalacetons hängen stark vom Methanolgehalt $\mathrm{ab}$, so daß Schwankungen im Methanolzusatz und Änderungen in der Methanolkonzentration während des Erwärmens einen bedeutenden Einfluß auf die Endfarbe haben (Abb. 2);

3. bei Gegenwart von Methanol strebt die Extinktion des Divanillalacetons keinem konstanten Endwert zu, sondern steigt stetig an (Abb. 3);

4. bei Methanolzusatz hängt die Endfarbe in erhöhtem Maße von der Reaktionstemperatur ab (Abb. 4).

Der Methanolzusatz stört die Reaktion des Acetons mit Vanillin so sehr, daß sie sich zur quantitativen Acetonbestimmung nicht eignet. Der Meinung von LINDBERG und ÖRSTRöM (7), daß die Methoden mit Salicylaldehyd und mit Vanillin gleich gut seien und sehr gut übereinstimmende Werte ergäben, müssen wir widersprechen. Diese Autoren haben nicht bei künstlicher Beleuchtung und nicht in Stickstoffatmosphäre gearbeitet und sie haben die langsame Einstellgeschwindigkeit des Gleichgewichts des Divanillalacetons und des Disalicylacetons beim Temperaturwechsel nicht beachtet.

Vanillin eignet sich zur Acetonbestimmung also nicht gleich gut wie Salicylaldehyd. Bei den günstigsten Vanillin- und KOH-Konzentrationen sind ohne Methanolzusatz die Extinktionen fast $4 \mathrm{mal}$ niedriger als mit der Salicylaldehydmethode. Unter Methanolzusatz laufen Sekundärreaktionen $\mathrm{ab}$, die die quantitative Bestimmung sehr stören.

\title{
Literatur
}

1. Nikırın, E. K., Žur. Obščej. Chim. 8, 2264 (1937). - 2. BäckSTRöm, H. L. J., Z. analyt. Chem. 123, 96 (1942). - 3. Levine, V. E. und M. Taterka, Clin. Chem. (New York) 3, 646 (1957). 4. BAHNER, F., Biochem. Z. 323, 327 (1952). - 5. BAHNER, F.,
Biochem. Z. 323, 318 (1952). - 6. Barner, F., Chemie-Ing.Techn. 25, 89 (1953). - 7. LindBerg, O. und A. Örström, Ark. Kem., Mineralog. Geol. Ser. A, 18/3, 1 (1945).

Professor Dr. med. F. Bahner

Endokrinolog. Abt. der Med. Poliklinik der Universität 69 Heidelberg, Hospitalstr. 3

\section{Mikropräparative Trennung der Lipidklassen von Milch und Blutserum}

\author{
Von G. CZEGLÉdi-Jankó \\ Aus dem Institut für Ernäbrungswissenschaft, Budapest, Ungarn (Direktor: Prof. Dr. R. Tarjän)
}

(Der Schriftleitung zugegangen am 16. März 1964)

\begin{abstract}
Zur präparativen Trennung der Hauptlipidklassen, in erster Linie in Milch und Blutserum wutde eine manuell gegossene, „dicke“ Kieselgelschicht angewendet. Mit dieser Methode konnten die einzelnen Hauptklassen in kurzer Zeit in solchen Mengen erhalten werden, daß die Fettsäurezusammensetzung selbst der Cholesterinesterfraktion der Milch mit einem katherometrischen Gaschromatographen ermittelt werden konnte.

Das qualitative Bild der getrennten Lipide und die Bestimmung der Fettsäurezusammensetzung der Lipidklassen lieferten einige neue Angaben hinsichtlich der Unterschiede zwischen Frauenmilch und Kuhmilch.

The preparative separation of the main classes of lipids, particularly in milk and blood serum, was performed on manually prepared "thick" layers of silica gel. The main lipid fractions of milk were obtained quickly and in sufficient quantities for a determination of the fatty acid composition, even in the cholesterol ester fraction, with a catherometric gas chromatograph.

The qualitative picture of the separated lipids and the establishment of the faity acid composition of the lipid classes yielded new data on the differences between human and cow's milk.
\end{abstract}

Für die klinische Diagnostik sind sowohl die Kenntnis der Zusammensetzung der Lipide als auch die Methoden zur Bestimmung der Komponenten von immer größerem Interesse. In einer seiner Mitteilungen betont SCHRADE
(1) die klinische Bedeutung der Trennung von Blutserumlipiden nach Verbindungsklassen und der Bestimmung der Fettsäurekomponenten in den einzelnen Lipidfraktionen. 
Zur Trennung von Lipiden nach Verbindungsklassen standen vorerst nur die säulenchromatographischen Methoden zur Verfügung. SCHRADE isolierte die freien Fettsäuren mittels Ionenaustauscher und trennte die Rückstände auf der chromatographischen Säule mit der Methode nach Lipsky (2). Hirsch und Ahrens (3) benutzten hierzu eine Kieselgelsäule. Diese Methode wurde von MANGord (4) als eine Standardmethode bezeichnet. Duncan und Garton $(5,6)$ zerlegten Rinderserumlipid auf der Kieselsäurekolonne zwecks Bestimmung der Fettsäurekomponenten in die einzelnen Lipidklassen. Menschliche Hautlipide wurden von BEY (7) gleichfalls auf der Kieselgelsäule getrennt. CAROLL (8) benutzte zur Zerlegung von Lipidklassen eine Florisilsäule. Hinsichtlich der Trennung von Lipiden nach Verbindungsklassen eröffnete die Dünnschichtchromatographie neue Wege. Den grundlegenden Forschungsarbeiten von Mangold (9), Malins (10) und KaufmanN (11), ferner der auf einem anderen Prinzip beruhenden dünnschichtchromatographischen Methode von WEICKER (12) folgend, veröffentlichten ZöllNer und Wolfram (13) ein Verfahren zur Trennung der Blutserumlipide. Einige Stoffwechsellipide untersuchte KaUfmanN (14) mittels der Dünnschichtchromatographie. In seiner Mitteilung gibt er einen Überblick über ähnliche Veröffentlichungen anderer Verfasser. Die Klassentrennung der Haar- und Hautlipide führte KaUfmanN (15) gleichfalls mittels Dünnschichtchromatographie durch.

Die dünnschichtchromatographische Trennung nach Lipidklassen ist bedeutend einfacher und rascher als die säulenchromatographische. Außerdem erhält man auf dem Dünnschichtchromatogramm ein viel vollständigeres Bild der Lipidklassen als auf der Säule, da die getrennten Verbindungsgruppen tatsächlich sichtbar sind und somit die Zusammensetzung der Lipidklassen unmittelbar qualitativ verglichen werden kann, was bei der Säulenchromatographie, die letzten Endes doch nur ein "Tasten im Dunkeln" ist, niemals erreicht werden kann. - Mit den dünnschichtchromatographischen Verfahren ist jedoch nicht jede Aufgabe einfach zu lösen. In den Frauenmilchlipiden beispielsweise beträgt der Cholesterinestergehalt etwa ein Hundertstel des Triglyzeridgehaltes; in einer, auf einer einzigen Schicht trennbaren Gesamtlipidmenge von $70-80 \mathrm{mg}$ ist also diese Klasse in so geringer Menge vorhanden, daß z. B. die gaschromatographische Bestimmung der Fettsäurekomponenten mit einem Gaschromatographen mittlerer Empfindlichkeit nicht immer gelingt und man gezwungen wäre entweder mit mehreren, oder mit sehr breiten Schichten nach ZöLLNER (13) zu arbeiten.

Auf Grund der neuesten Resultate in der Dünnschichtchromatographie haben wir ein 'Trennverfahren entwickelt, nach der auf eine einzige Schicht größere Mengen des Gesamtlipids aufgetragen und somit getrennt werden können, wodurch auch die in geringen Quantitäten vorhandenen Klassen in solcher Menge zu erhalten sind, $\mathrm{da} \beta$ die gaschromatographische Bestimmung der Fettsäuren ermöglicht wird.
Trennung nach Verbindungsklassen auf der „dicken" Kieselgelschicht

Zur mikropräparativen Trennung der Lipidklassen in Milch- und Blutserum haben wir eine mit Hand gegossene, 1-3 $\mathrm{mm}$ dicke Kieselgelschicht verwendet, die in gewissem Sinne den von PAstuska (16) benutzten borsauren Kieselgelschichten glich; die getrennten Klassen wurden durch Elution im Chromatographierrohr isoliert. Die zu trennende Lipidmenge ist durch die Empfindlichkeit der Methode bestimmt. Bei Frauenmilch z. B. benötigt man etwa bis $500 \mathrm{mg}$, um die Fettsäurekomponenten sämtlicher Hauptlipidklassen mit einem Gaschromatographen von mittlerer Empfindlichkeit bestimmen zu können. Durch die Anwendung der „dicken“ Kieselgelschicht konnten selbst vom Cholesterinester etwa $1 \mathrm{mg}$ erhalten werden.

Die quantitative Bestimmung der getrennten Klassen, soweit es die Größenordnung erlaubte, wurde gravimetrisch durchgeführt; zur Bestimmung von kleinen Mengen haben wir jedoch die üblichen photometrischen Methoden herangezogen.

Bei der Verwendung verschiedener Fließmittel zur Entwicklung der Schichtchromatogramme beobachteten wir, daß die Charakterisierung des Fließmittels durch den Siedepunktbereich nicht immer genügend ist. Der $R_{F}$-Wert der Flecke z. B. verändert sich bei Kohlenwasserstoffgemischen von gleichem Siedepunktbereich, in Abhängigkeit vom Verhältnis der einzelnen Komponenten verschiedenen Siedepunktes, besonders aber vom Verhältnis der verschiedenen Isomere. Wenn man ständig mit derselben Mischung arbeitet, fällt dieser Umstand nicht auf, doch wird die Reproduzierbarkeit der Methode eingeschränkt. Eine solche Ungewißheit kann vermieden werden, wenn als Kohlenwasserstoffkomponente des Fließmittels eine einheitliche Substanz, z. B. $\mathrm{n}$-Hexan angewendet wird. Unsere Beobachtungen gehen dahin, $\mathrm{da}$ es noch viel vorteilhafter ist, das Gemisch einer größeren Menge von n-Heptan und einer kleineren, oder höchstens derselben Menge von Petroläther (Sp. 40-60 $0^{\circ}$ zu benutzen. Petroläther unterschiedlicher Zusammensetzung, wenn nicht im Ubergewicht, beeinflußt den $R_{\mathrm{F}}$-Wert nicht entscheidend, macht jedoch die Trennung selektiver. Durch Veränderung der Proportion dieser beiden Komponenten kann die weitere Regelung der $R_{\mathrm{F}}$-Verhältnisse verwirklicht werden. So konnte z. B. ein solches Fließmittel gefunden werden, das es erlaubte das Cholesterin und die Mono- bzw. Diglyzeridklasse ungetrennt zu erhalten. Die quantitative Bestimmung der beiden Klassen kann auch bei gemeinsamer Elution ungestört nebeneinander durchgeführt werden. Gleichfalls wurden Glyzerylätherdiester mit den Triglyzeriden zusammen erhalten und eluiert. In diesem Sinne werden wir also im weiteren manchmal anstatt „Klasse“ auch den Ausdruck „Zone" benutzen. Die Zonen können nötigenfalls in Klassen getrennt werden. Es sei bemerkt, daß wenn man anstatt Petroläther das einheitliche n-Pentan mit n-Heptan vermischt, die Trennung bei weitem nicht so selektiv verläuft. - Das Fließmittel bestehend aus n-Heptan und Petroläther ist dem Gemisch von Benzol und Petroläther nach KaufmanN (14), d. h. der Mischung eines Kohlenwasserstoffes von höherem und eines von niederem Siedepunkt ähnlich.

Auf dem entwickelten Chromatogramm haben wir die Lage der getrennten Lipidzonen auf die gewohnte Weise durch Sichtbarmachung von Markierungsflecken bestimmt. Um die Erwärmung der ganzen Schicht zu vermeiden, haben wir diese Flecke mittels eines Fadenofens erwärmt. Der Fadenofen ist eine in Glasrohr eingelegte Heizspirale, die ausschließlich die unmittelbar unter ihr liegende Fläche erwärmt. - Da Joddämpfe 
aus der verhältnismäßig großen Masse der „dicken“ Kieselgelschicht bei Zimmertemperatur nur schwer restlos beseitigt werden können, haben wir, wenn zur Sichtbarmachung der Markierungsflecke das Joddampfverfahren herangezogen wurde, den übrigen Teil der Schicht mit Glasscheiben abgedeckt.

Die getrennten Lipidzonen wurden aus dem abgeschabten und zerriebenen Kieselgel in einem kleinen Chromatographierrohr mit Chloroform Methanol (1:1) eluiert. Da polare Lösungsmittel auch Gips in meßbarer Menge lösen, war bei der Bestimmung des $\mathrm{Ge}$ wichts der isolierten Lipidzonen eine Korrektion unumgänglich. - Von den getrennten Lipidklassen bzw. Zonen der Frauenmilch wurden die Cholesterinester, das Cholesterin und die Phospholipide kolorimetrisch bestimmt. Aus dem kolorimetrisch bestimmten Gehalt an Cholesterin in Esterbindung wurde die Menge des Cholesterinesters selbst nach Multiplikation mit 1,5 berechnet. Da die Phospholipide an der Startstelle zurückbleiben, können sie schon vor dem Besprühen mit Phophormolybdänsäure abgeschabt werden, wodurch ihre Verunreinigung mit Phosphor vermieden werden kann. Die Menge des Phospholipids wurde aus dem Phosphorgehalt durch Multiplikation mit 25 berechnet. - Die anderen Zonen wurden gravimetrisch bestimmt, bei den Mono- bzw. Diglyzeriden wurde die kolorimetrisch bestimmte Cholesterinmenge in Abzug gebracht.

$\mathrm{Zu}$ den gaschromatographischen Untersuchungen haben wir die Umesterung der Fettsäuren mit der Methode von CRAIG (17) durchgeführt. Die erhaltenen Methylester wurden auf der Kieselgelschicht getrennt und eluiert. Hinsichtlich der quantitativen Bewertung der Gaschromatogramme sind die Ansichten verschieden (18). Wir haben die Menge der Methylester aus der „peak area" in Prozenten der bewerteten gesamten Menge berechnet.

\section{Versuche}

Extraktion der Lipide nach Broor (19), modifiziert von KAUFMANN (20). Milch oder Blutserum wird unter ständigem Schütteln einem 15-20 fachen Volumen Äthanol-Diäthyläther-Gemisch (3:1) tropfenweise zugesetzt. Die Mischung bringt man für eine Stunde in eine Schüttelmaschine und läßt dann über Nacht in Stickstoffatmosphäre stehen; die ausgefällten Eiweißstoffe werden sodann filtriert und mit Äther-Petroläther gewaschen. Die Waschflüssigkeit wird mit dem lipidhaltigen äthanol-ätherischen Filtrat vereint und das Gemisch unter vermindertem Druck in Stickstoffatmosphäre eingedampft. Den Rest verreibt man mit wenig wasserfreiem $\mathrm{Na}_{2} \mathrm{SO}_{4}$, nimmt mit Petroläther auf und verdampft das Lösungsmittel im Stickstoffstrom unter vermindertem Druck. Den trockenen Rückstand läßt man über Nacht in einem Exsikkator über $\mathrm{P}_{2} \mathrm{O}_{5}$ stehen und wägt. - Es ist ratsam zur Extraktion kein Methanol zu verwenden, da in diesem Fall auf dem Chromatogramm Methylester-Artefakten erscheinen können.

\section{Qualitative DC-Trennung nach Lipidklassen}

a) $30 \mathrm{~g}$ Kieselgel G „Merck“ wird mit $60 \mathrm{ml}$ dest. Wasserkörnchenund luftblasenfrei verrieben und mit einem „Desaga“-Streicher ${ }^{1}$ ) auf eine $20 \times 20 \mathrm{~cm}$ Glasscheibe gestrichen. Die getrocknete

1) Fa. Desaga GmbH, Heidelberg.
Platte wird bei $105^{\circ} 10 \mathrm{Min}$. aktiviert und bis zur Verwendung in einem Raum von $10 \%$ rel. Feuchtigkeitsgehalt aufbewahrt.

b) Zur Entwicklung des Chromatogrammes werden aus der Benzol-ítösung des Lipids, die pro Milliliter $100 \mathrm{mg}$ Substanz enthält, $1-5 \mu l(100-500 \mu \mathrm{g})$ entlang eines $8 \mathrm{~mm}$ langen Striches auf die Kieselgelschicht aufgetragen. Fließmittel: $65 \mathrm{~m} l \mathrm{n}$-Heptan, $20 \mathrm{~m} l$ Petroläther (Sp. $40-60^{\circ}$ ), $15 \mathrm{ml}$ Diäthyläther, $1 \mathrm{ml}$ Eisessig. Temperatur: $20-24^{\circ}$, Laufstrecke: $14-16 \mathrm{~cm}$.

c) Zur Sichtbarmachung des Chromatogramms wird die getrocknete Schicht mit einer 10proz. alkoholischen Phosphormolybdänșäurelösung besprüht und bei $120^{\circ}$ bei $10-15 \mathrm{Min}$. getrocknet. Anstatt Phosphormolybdänsäure kann auch das Joddampfverfahren angewendet werden.

Präparative Trennung nach Lipidzonen auf der "dicken" Kieselgelschicht a) Je nach Menge des zu trennenden Lipids werden 16-64 g Kieselgel G "Merck" und 2-8 g Alabastergips vermischt, mit $20-120 \mathrm{~m} l$ dest. Wasser in einem Mörser körnchen- und luftblasenfrei verrieben. Der Brei wird auf eine mit Azeton entfettete $20 \times 20 \mathrm{~cm}$ Glasscheibe gegossen und mit der Mörserkeule möglichst gleichmäßig ausgestrichen. Dann wird die mit der einen Hand fixierte, genau horizontal liegende Platte mit der anderen Hand angeklopft. Durch die Vibration verteilt sich der Brei gleichmäßig. Der ganze Vorgang soll nicht mehr als 1-1,5 Min. in Anspruch nehmen. Die über Nacht an derLuft getrockneten Platten werden bei $110^{\circ} 45 \mathrm{Min}$. lang aktiviert und bis zur Verwendung in einem Raum von $10 \%$ rel. Feuchtigkeitsgehalt aufbewahrt. (Ohne Gipszusatz hergestellte Schichten erhalten während des Trocknens Sprünge!)

b) Zur Entwicklung des Chromatogramms werden aus der Benzol-Lösung des Lipids, die pro Milliliter $200 \mathrm{mg}$ Substanz enthält, bis zu $2500 \mu l$ in drei, je $45 \mathrm{~mm}$ langen Strichen (Abb. 1), nebst vier Markierungsflecken ( $x$ ) aufgetragen. Sollte wenig zu trennendes Material vorhanden sein, kann man zur Markierung eine fremde Substanz verwenden. Bei Milchlipiden ist es angezeigt an den beiden Seiten auch Blutserumlipid zur Markierung aufzutragen $(\uparrow)$, um auf dem Chromatogramm die Lage der Cholesterinester genauer markieren zu können. Fließmittel: $60 \mathrm{ml}$ $n$-Heptan, $60 \mathrm{~m} l$ Petroläther (K.p. $40-60^{\circ}$ ), $20 \mathrm{~m} l$ Diäthyläther, $1,5 \mathrm{~m} l$ Eisessig. Temperatur: $20-24^{\circ}$, Laufstrecke: $14-16 \mathrm{~cm}$.

c) Zu präparativen $Z$ wecken werden bei „dicken“ Schichten nur die vier Markierungsstreifen entwickelt, indem man das Sprühreagens bei Anwendung einer Maske aufbringt (Abb. 2). Die Markierungschromatogramme werden mit einem Fadenofen erwärmt, dessen Heizspannung $24 \mathrm{~V}(70 \mathrm{~W})$ beträgt. Bei Anwendung von Joddampf werden den nicht sichtbar zu machenden Teilen der Schicht Glasscheiben dicht angelegt (Abb. 3).

d) Zwecks Elution schabt man die markierten Zonen ab, zerreibt sie und bringt sie in ein Chromatographierohr von $1 \mathrm{~cm}$ Durchmesser. Die Elution geschieht mit einer Chloroform-MethanolMischung (1:1). Zur vollständigen Elution benötigt man $80 \mathrm{ml}$ Lösungsmittel (Abb.4). Das Eluat wird vom Lösungsmittel unter vermindertem Druck im Stickstoffstrom befreit, der Rückstand über Nacht im Vakuum über $\mathrm{P}_{\mathbf{2}} \mathrm{O}_{5}$ getrocknet, wonach die quantitative Bestimmung durchgeführt werden kann. Wenn dies gravimetrisch geschieht, mißt man vor dem Abschaben die Höhe der Zone und das Meßresultat wird mit dem, der Höhe entsprechenden Blindwert korrigiert. Der Blindwert muß bei jedem KieselgelGips-Gemisch und bei jeder verwendeten Schichtdicke bestimmt werden.

\section{Die Umesterung und Reinigung der Fettsäuren}

a) $\mathrm{Zu}$ höchstens $50 \mathrm{mg}$ Lipid werden $5 \mathrm{ml}$ abs. Methanol und $0,5 \mathrm{ml}$ methanolisches Natriummethylat $(0,5 \mathrm{~g}$ metallisches Natrium/l abs. Methanol) gegeben und das Gemisch wird eine halbe Stunde im Stickstoffstrom am Rückfluß gekocht. Nach Abkühlen setzt man der Lösung $0,1 \mathrm{~m} l$ Eisessig zu und verdampft das Lösungsmittel unter vermindertem Druck im Stickstoffstrom.

b) Zwecks Reinigung des Methylesters wird das esterisierte Lipid in einem Mengenverhältnis von etwa $5 \mathrm{mg} / \mathrm{cm}$, nebst den 


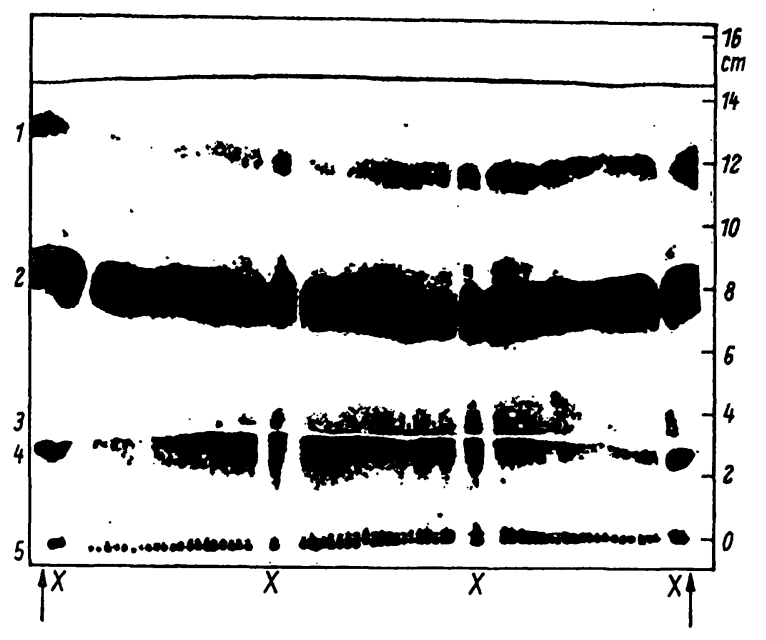

Abb. 1

Zonentrennung des Frauenmilchlipids auf einer „dicken“ Kieselgelschicht. Die Zonen:

1. Cholesterinester

2. Triglyzeride, darüber unmittelbar Glyzerylätherdiester

3. Freie Fettsäuren

4. Cholesterin sowie Di- und Monoglyzeride

5. Phospholipide

Das ganze Chromatogramm wurde nur für demonstrative Zwecke sichtbar gemacht, ansonsten sind nur die Markierungschromatogramme $(X$ und $\uparrow)$ zu sehen.

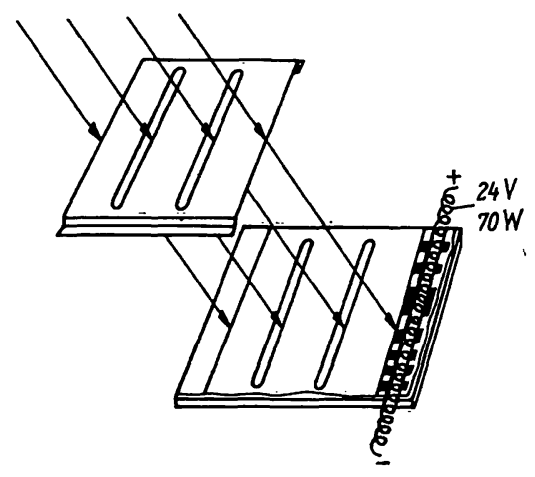

Abb. 2

Besprühung der Markierungschromatogramme mit dem Sprühreagens bei Anwendung einer Maske unmittelbat auf der Platte; Sichtbarmachung nach Besprühung mittels Fadenofen

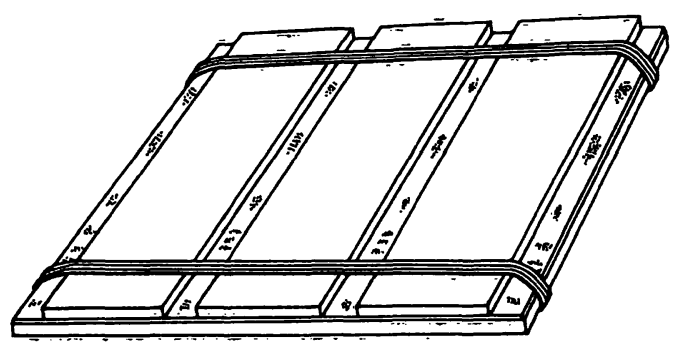

Abb. 3

Sichtbarmachung der Markierungschromatogramme einer „dicken“" Kieselgelschicht mittels Joddämpfen

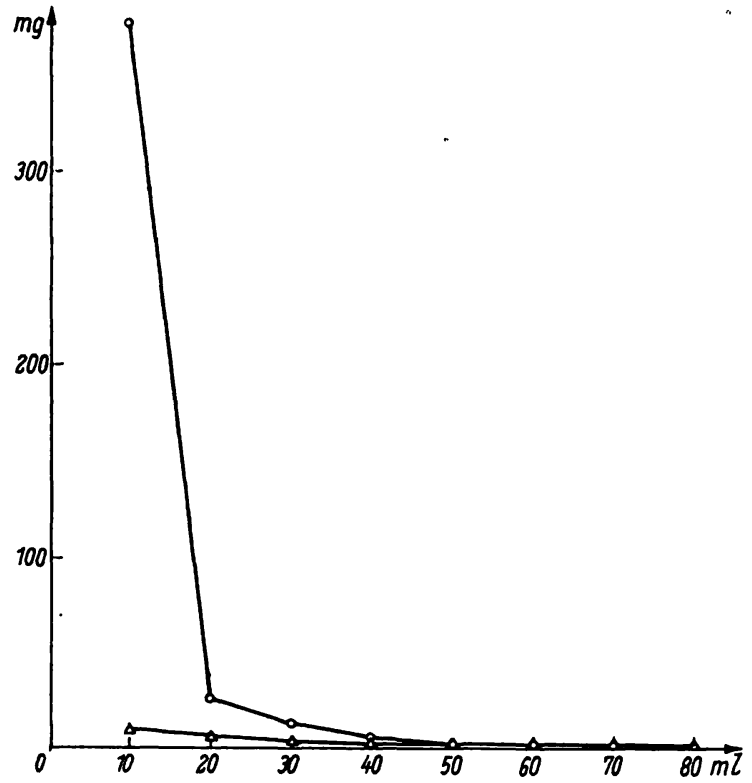

Abb. 4

Elutionsdiagramme der von "dicken" Schichten abgeschabten Lipidzonen: a) Triglyzeridzone des Frauenmilchlipids; b) Phospholipidzone des Blutserumlipids

Markierungsflecken strichförmig auf die wie beschrieben hergestellte Schicht aufgetragen. Sodann entwickelt man gemäß Punkt b) das Chromatogramm und macht die Markierungsflecke sichtbar. Die fettsauren Methylester erscheinen zwischen den Cholesterinester- und Triglyzeridflecken und werden aus der abgeschabten Schicht isoliert.

Gascbromatographische Untersucbung der fettsauren Metbylester

Zur Untersuchung der fettsauren Methylester kann jedes, diesem Zweck entsprechende gaschromatographische Gerät und Verfahren benutzt werden. Die vorliegenden Untersuchungen wurden mit der nachstehenden Einrichtung durchgeführt:

Getät: »Aerograph A-90-P“c

Kolonne: $\quad 10 \mathrm{Fu}, 1 / 4 \varnothing$; Füllung: Chromosorb W 3060 mesh, $15 \%$ DEGS.

Trägergas: $\mathrm{H}_{2}, 60 \mathrm{ml} / \mathrm{Min}$.

Heizstrom: $200 \mathrm{~mA}$; Attenuator: 1

Temperatur: Kolonne $180^{\circ}$; Detektor $240^{\circ}$; Injektor $220^{\circ}$; Kollektor $215^{\circ}$;

Papierförderung: $1 / 6 / \mathrm{Min}$.

Menge der Probe: 0,5-1,0 $\mu$ l.

\section{Ergebnisse}

Nachfolgend werden die Resultate je einer Trennung der Lipidklassen und der Bestimmung ihrer Fettsäurekomponenten in Blutserum, Frauenmilch und Kuhmilch beschrieben.

Die Abbildung 5 veranschaulicht das qualitative Bild der dünnschichtchromatographisch getrennten Lipidklassen in den erwähnten drei biologischen Substanzen. Die Klassen sind folgende:

1. Cholesterinester

2. Glyzeryläther-diester

3. Triglyzeride

4. Freie Fettsäuren

5. Cholesterin
6. Di- und Monoglyzeride, mit nicht identifizierten Subfraktionen

7. Phospholipide. 


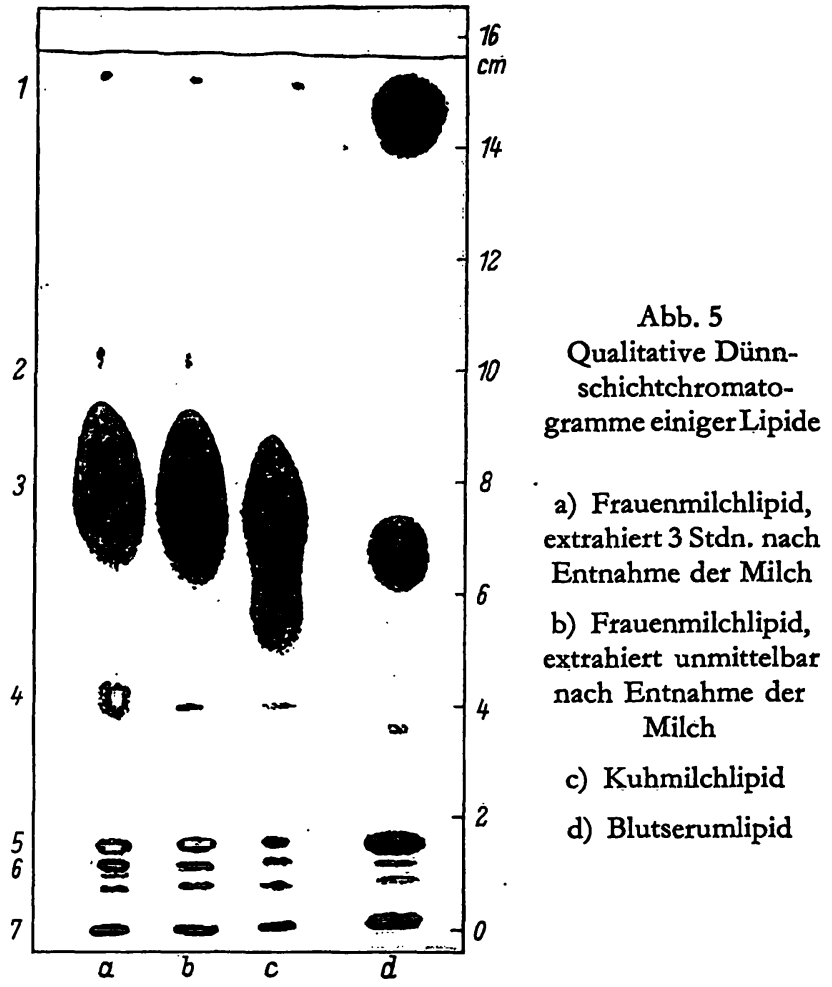

Die Farbenintensität der Flecke bei verschiedenen Verbindungsarten ist der Menge der Substanz nicht proportional, da die Verfärbung eher von der chemischen Natur der Substanz abhängig ist. Aus der Dimension der einzelnen Flecke ist es jedoch sichtbar, daß die Lipidklassen im Blutserumcbromatogramm am gleichmäßigsten verteilt sind. Bei den Milcblipiden ist die Triglyzeridfraktion die größte. Die Triglyreridflecke in der Frauenmilch und in Kubmilch weisen eine auffallende Verschiedenbeit auf. Die in der Frauenmilch sind einheitlicher, dagegen die in der Kuhmilch eher auseinandergezogen. Letztere weisen eine deutliche Subfraktion auf. Diese Erscheinung zeigt, daß der Aufbau der Triglyzeride in der Frauenmilch weniger mannigfaltig ist als in der Kuhmilch.

Auch in den freien Fettsäuren der beiden Milcharten zeigt sich ein wesentlicher Unterschied. Das Frauenmilchlipid enthält, selbst unmittelbar nach Entnahme, mehr freie Fettsäuren (Abb. $5 \mathrm{~b}$ ) als das nach mehrstündigem Stehen extrahierte Kuhmilchlipid (Abb. 5c). Für drei Stunden bei einer Temperatur von $+5^{\circ}$ aufbewahrt, steigt in der Frauenmilch die Menge der freien Fettsäuren weiter an (Abb. 5a). Dies zeigt, daß in der Frauenmilch, infolge der größeren Lipaseaktivität, die Fettsäuren leicht aus der Esterbindung befreit werden. Diese Erscheinung steht, aus ernährungsphysiologischer Sicht, mit den Beobachtungen von DrOESE (21) in Einklang, der mit seinen Mitarbeitern feststellte, daß Neugeborene und besonders Frühgeborene die Lipide der eine geringere Enzymaktivität aufweisenden Kuhmilch so lange nur schwer verdauen können, bis die eigene Pankreas- und Darmlipaseproduktion ein gewisses Niveau erreicht hat.

Die Resultate der quantitativen Bestimmung der einzelnen Lipidklassen sind in Tabelle 1, die der gaschro- matographischen Fettsäurebestimmungen in Tabelle 2,3 , und 4 zusammengefaßt. Hieraus ist zu ersehen, daß die Kubmilch über das reichste Fettsäurespektrum verfügt (Abb. 6). Unter den gegebenen Untersuchungsbe-

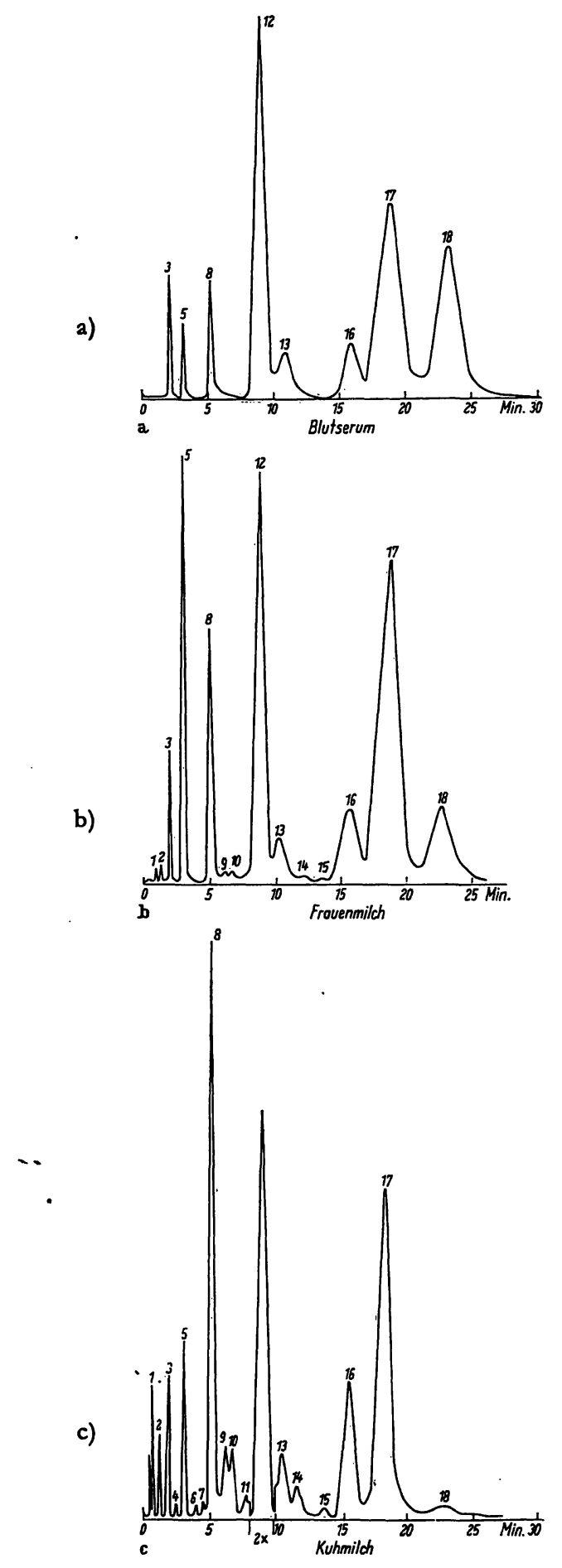

Abb. 6

Gaschromatogramm der Fettsäuren in den Gesamtlipiden von Blutserum (a), Frauenmilch (b) und Kuhmilch (c). Identifizierte $\begin{array}{lcl}1: C_{4}, C_{6} & 7: C_{13} & 13: C_{18} \\ 2: C_{8} & 8: C_{14} & 14: C_{17} \\ 3: C_{10} & 9: C_{14} & 15: C_{17}(?) \\ 4: C_{11} & 10: C_{15} & 16: C_{18} \\ 5: C_{12} & 11: C_{16}(?) & 17: C_{18} \\ 6: C_{12} & 12: C_{16} & 18: C_{18}\end{array}$ Identifizierung durch Bezugssubstanzen (kursiv) bzw. durch Berechnung aus den Retentionszeiten 
dingungen konnten 18 Fettsäuren aus der Kuhmilch differenziert werden. In der Frayenmilch wurden 14, im Blutserumlipid unter gleichen Verhältnissen nur 8 Fettsäuren nachgewiesen. Obzwar Graser (22) mit einem empfindlicheren Gaschromatographen im Blutserum 20 verschiedene Fettsäuren nachweisen konnte, zeigen doch unsere Ergebnisse, daß die Fettsäurezusammensetzung der drei untersuchten Lipide unterschiedlich ist. In diesem Zusammenhang sei betont, daß zum Vergleich der Fettsäurezusammensetzung der verschiedenen Lipide und Lipidklassen nur die unter identischen Untersuchungsbedingungen erhaltenen Daten herangezogen werden können. Dies ist der Grund dafür, daß wir unsere Ergebnisse bezüglich der oben erwähnten Lipidarten nicht eingehender mit den in den wichtigsten diesbezüglichen Mitteilungen (23-30) veröffentlichten Daten verglichen haben.

Tab. 1

Quantitative Verteilung der Hauptlipidklassen in Frauenmilch-, Kuhmilch- und Blutserumlipiden. Werte in $\mathbf{g}$

\begin{tabular}{|c|c|c|c|c|c|c|c|c|}
\hline & $\begin{array}{l}\text { Aufgetragene } \\
\text { Lipid- } \\
\text { menge }\end{array}$ & $\begin{array}{c}\text { Cholesterin- } \\
\text { ester }^{2} \text { ) }\end{array}$ & $\begin{array}{c}\text { Triglyzeride } \\
\text { (Frauenmilch: + } \\
\text { Glyzeryläther- } \\
\text { diester) })^{1} \text { ) }\end{array}$ & $\begin{array}{l}\text { Freie Fett- } \\
\text { säuren }{ }^{1} \text { ) }\end{array}$ & $\begin{array}{l}\text { Chole- } \\
\text { sterin }^{2} \text { ) }\end{array}$ & $\begin{array}{c}\text { Mono- + Digly- } \\
\left.\text { zeride }^{3}\right)\end{array}$ & $\begin{array}{l}\text { Phospho- } \\
\text { lipide }^{2} \text { ) }\end{array}$ & $\begin{array}{l}\text { Rückgewin- } \\
\text { nung }\end{array}$ \\
\hline $\begin{array}{l}\text { Frauenmilch- } \\
\text { lipid }\end{array}$ & 0,5000 & 0,0075 & 0,4312 & 0,0148 & 0,0400 & 0,0140 & 0,0250 & $106,5 \%$ \\
\hline $\begin{array}{l}\text { Kuhmilch- } \\
\text { lipid }\end{array}$ & 0,5000 & - & 0,4862 & - & - & - & - & \\
\hline $\begin{array}{l}\text { Blutserum- } \\
\text { lipid }\end{array}$ & 0,0600 & 0,0180 & $\left.0,0151^{1}\right)$ & 0,0029 & 0,0040 & 0,0017 & 0,0150 & $94,5 \%$ \\
\hline
\end{tabular}

1) Gravimetrisch ermittelt

2) Kolorimetrisch ermittelt

3) Gesamtmenge gravimetrisch, Cholesterin kolorimetrisch ermittelt und in Abzug gebracht

Tab. 2

Fettsäureesterzusammensetzung der Blutserumlipide nach Verbindungsklassen von $C_{10}$ bis $C_{18}$ (dopp. unges.) in Prozenten

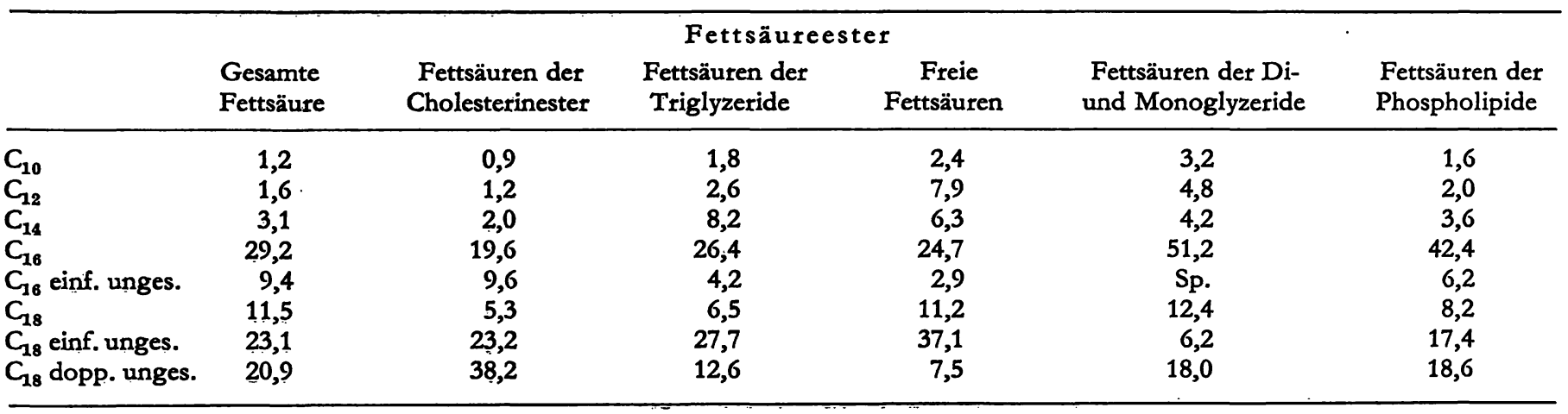

Tab. 3

Fettsäureesterzusammensetzung der Frauenmilchlipide nàch Verbindungsklassen von $C_{10}$ bis $C_{18}$ (doppelt unges.) in Prozenten

\begin{tabular}{|c|c|c|c|c|c|c|}
\hline \multicolumn{7}{|c|}{ Fettsäureester } \\
\hline & $\begin{array}{l}\text { Gesamte } \\
\text { Fettsäure }\end{array}$ & $\begin{array}{l}\text { Fettsäuren der } \\
\text { Cholesterinester }\end{array}$ & $\begin{array}{l}\text { Fettsäuren der } \\
\text { Triglyzeride }\end{array}$ & Freie Fettsäuren & $\begin{array}{l}\text { Fettsäuren der Di- } \\
\text { und Monoglyzeride }\end{array}$ & $\begin{array}{l}\text { Fettsäuren der } \\
\text { Phospholipide }\end{array}$ \\
\hline $\begin{array}{l}C_{6} \\
C_{8} \\
C_{10} \\
C_{12} \\
C_{14} . \\
C_{14} \text { einf. unges. } \\
C_{15} \\
C_{16} \\
C_{16} \text { einf. unges. } \\
C_{17} . \\
C_{18} \\
C_{18} \text { einf. unges. } \\
C_{18} \text { dopp. unges. }\end{array}$ & $\begin{array}{r}\text { Sp. } \\
\text { Sp. } \\
1,4 \\
5,2 \\
8,1 \\
\text { Sp. } \\
0,2 \\
27,4 \\
4,3 \\
\text { Sp. } \\
8,3 \\
35,2 \\
10,2\end{array}$ & $\begin{array}{l}\text { Sp. } \\
\overline{1,6} \\
5,6 \\
3,0 \\
= \\
\overline{8,6} \\
2,0 \\
\overline{9,0} \\
33,4 \\
36,8\end{array}$ & $\begin{array}{r}\text { Sp. } \\
\text { Sp. } \\
2,0 \\
4,2 \\
8,0 \\
\text { Sp. } \\
0,2 \\
27,6 \\
3,4 \\
\text { Sp. } \\
8,8 \\
36,0 \\
9,8\end{array}$ & $\begin{array}{r}\overline{-} \\
2,4 \\
7,9 \\
6,3 \\
\overline{-} \\
24,7 \\
2,9 \\
\overline{11,2} \\
27,1 \\
17,5\end{array}$ & $\begin{array}{r}- \\
\overline{0} \\
3,6 \\
3,6 \\
6,0 \\
\overline{-} \\
22,2 \\
5,3 \\
\overline{17,7} \\
35,3 \\
9,3\end{array}$ & $\begin{array}{r}\overline{-} \\
2,0 \\
7,4 \\
6,2 \\
\overline{-} \\
22,0 \\
3,4 \\
16,2 \\
24,6 \\
18,2\end{array}$ \\
\hline
\end{tabular}


Tab. 4

Prozentuale Fettsäurcesterzusammensetzung der Kuhmilchlipide nach Verbindungsklassen von $C_{4}$ bis $C_{18}$ (doppelt unges.)

\begin{tabular}{|c|c|c|c|c|c|c|}
\hline \multicolumn{7}{|c|}{ Fettsäureester } \\
\hline & $\begin{array}{l}\text { Gesamte } \\
\text { Fettsäure }\end{array}$ & $\begin{array}{l}\text { Fettsäuren der } \\
\text { Cholesterinester }\end{array}$ & $\begin{array}{l}\text { Fettsäuren der } \\
\text { Triglyzeride }\end{array}$ & Freie Fettsäure & $\begin{array}{l}\text { Fettsäuren der Di- } \\
\text { und Monoglyzeride }{ }^{1} \text { ) }\end{array}$ & $\begin{array}{l}\text { Fettsäuren der } \\
\text { Phospholipide }^{1} \text { ) }\end{array}$ \\
\hline$C_{4,6}$ & 5,2 & - & 8,4 & - & - & - \\
\hline$C_{8}$ & 1,2 & 一 & 1,0 & - & - & - \\
\hline$C_{10}$ & 3,6 & 3,1 & 3,4 & 4,0 & Sp. & - \\
\hline$C_{11}$ & Sp. & - & Sp. & - & - & - \\
\hline $\mathrm{C}_{12}$ & 5,6 & 5,1 & 5,0 & 5,6 & 3,1 & 6,0 \\
\hline$C_{12}$ einf. unges. & 0,1 & - & 0,1 & - & - & - \\
\hline$C_{13}$ & 0,1 & - & 0,2 & - & - & - \\
\hline $\mathrm{C}_{14}$ & 10,6 & 14,2 & 10,4 & 8,1 & 7,2 & 6,1 \\
\hline$C_{14}$ & 1,2 & $\div$ & 1,0 & Sp. & Sp. & - \\
\hline$C_{15}$ & 1,5 & - & 1,6 & - & - & - \\
\hline $\mathrm{C}_{16}$ & 0,4 & - & 0,4 & - & - & - \\
\hline$C_{16}$ einf. unges. & 33,2 & 30,2 & 31,0 & 30,1 & 30,3 & 28,4 \\
\hline$C_{16}$ & 1,0 & 4,7 & 1,4 & 1,9 & 8,0 & 4,1 \\
\hline$C_{17}$ & 0,2 & - & 0,6 & - & - & - \\
\hline$C_{17}$ einf. unges. & Sp. & - & Sp. & - & - & - \\
\hline$C_{18}$ & 10,8 & 6,8 & 9,8 & 10,0 & 5,4 & 9,6 \\
\hline$C_{18}$ einf. unges. & 23,7 & 20,1 & 24,5 & 26,3 & 23,6 & 30,0 \\
\hline $\mathrm{C}_{18}$ dopp. unges. & 1,5 & 4,2 & 1,2 & 2,4 & 10,8 & 4,2 \\
\hline
\end{tabular}

1) Berechnet in Prozenten des Gesamtlipids

\section{Literatur}

1. Schrade, W., E. Böhle und R. Biegler, Fette Seifen einschl. Anstrichmittel 62, 673 (1960). - 2. LipSKY, S. R., A. HAAvix, C. L. Hopper und R.W. McDivitr, J. Clin. Invest. 36, 233 (1957). - 3. Hirsch, J. und E. H. Ahrens jr., J. biol. Chemistry 233, 311 (1958). - 4. Mangord, H. K., Aliphatische Lipide in E. StahL, Dünnschichtchromatographie, S. 152, Springer-Verlag, Berlin-Göttingen-Heidelberg (1962). - 5. DunCAN, W. R. H. und G. A. Garton, J. Lipid Res. 3, 53 (1962). - 6. Duncan, W. R. H. und G. A. Garton, Biochem. J. 89, 414 (1963). - 7. BeY, K., Fette Seifen einschl. Anstrichmittel 65, 611 (1963). - 8. Caroll, K. K.; J. Lipid Res. 2, 135 (1961). - 9. Mangold, H. K. und D. C. Marins, J. Amer. Oil Chemist's Soc. 37, 383 (1960). 10. Malins, D. C. und H. K. Mangold, J. Amer. Oil Chemist's Soc. 37, 576 (1960). - 11. Kaufmann, H. P., Z. Makus und B. DAs, Fette Seifen einschl. Anstrichmittel 63, 807 (1961). - 12. WeICKer, H., Klin. Wschr. 37, 763 (1959). - 13. ZöLLNER, N. und G. Wolfram, Klin. Wschr. 40, 1098 (1962). - 14. KaupmanN, H. P. und C. V. Viswanathan, Fette Seifen einschl. Anstrichmittel 65, 538 (1963). - 15. Kaufmann, H. P. und C. V. Viswanathan, Fette Seifen einschl. Anstrichmittel 65, 607 (1963). - 16. Pastus-
KA, G., Z. analyt. Chem. 179, 427 (1961). - 17. CraIG, B. M. und N. L. Murty, J. Amer. Oil Chemist's Soc. 37, 549 (1959). 18. Kaupmann, H. P. und G. Mankel, Fette Seifen einschl. Anstrichmittel 65, 179 (1963). - 19. BlooR, W. R., J. biol. Chemistry 77, 53 (1928). - 20. KaufmanN, H. P. und H. GarLOFF, Fette Seifen einschl. Anstrichmittel 62, 679 (1960). - 21. Droese, W. und H. Stollex, Fette Seifen einschl. Anstrichmittel 62, 281 (1960). - 22. Glaser, A., G. Grimmer, E. Jantzen und H. Oertel, Biochem. Z. 336, 274 (1962). - 23. American Academy of Pediatrics - Report, Pediatrics 26, 1039 (1960). 24. Gander, W., R. G. Jensen und J. Sampugna, J. Dairy Sci: 45, 323, (1962). - 25. HAAB, W., L. M. SMTTH and E. L. JACK, J. Dairy Sci. 42, 454 (1959). - 26. Herb, S. F., P. Magrdman, F. E. Luddy und R. RIEMEnschneider, J. Amer. Oil Chemist's Soc. 39, 142 (1962). - 27. Insule, W. und E. H. Ahrens, Biochem. J. 72, 27 (1959). - 28. KaufmanN, H. P. und G. MankeL, Fette Seifen einschl. Anstrichmittel 65, 295 (1963). - 29. MAGrDMaN, P., S. F. HERb, R.A. Barford und R. W. RIEMENSCHNEIDER, J. Amer. Oil Chemistr's Soc. 39, 137 (1962). - 30. Pieraccini, P., M. Morgantini, Riv. clin. pediatr. Firenze 68, 241; 386, (1961).

Dr. G. Czeglédi-Jankó Institut für Ernährungswissenschaft Budapest IX (Üngarn) Gyali ut 3/a 